\title{
Area MT+ in Human Brain Responsible for Optic Flow Processing
}

\author{
Jieyu Fan ${ }^{1}$ \\ ${ }^{1}$ Tung Wah High School of Dongguan City, Dongguan, Guangdong 523808, China
${ }^{*}$ Corresponding author.1105529818@qq.com
}

\begin{abstract}
Optic flow - the dynamic light motion pattern that projects on moving observers' retina - plays great roles in many behaviors. Huge neurophysiological and neuroimaging studies have been conducted to reveal the cortical areas in human brain that respond to optic flow. One of the most important cortical areas is area MT+ (MT \& MST). In the current paper, I tried to summarize the previous studies about human area MT+ and pointed out their existing questions. Based on these questions, I proposed several future research directions. Solving these questions will improve our understanding about the role of area MT+ in optic flow processing.
\end{abstract}

Keywords: MT+, MST, MT, human brain, optic flow

\section{INTRODUCTION}

When observers are moving in the environment, the objects in the environment would project on observers' retina and generate a dynamic motion pattern named as optic flow. This concept was first introduced by James J. Gibson (1950), an American psychologist. Optic flow contains rich information, such as the 2D structure information - focus of expansion (FoE) and the 3D structure information, e.g., speed gradient or motion parallax (e.g., Koenderink, 1986; Longuet-Higgins \& Prazdny, 1980).

According to the definition of optic flow, it is clear that optic flow is generated by our self-movements. In our daily life, the self-movements are various. For example, while walking on a street, we would like to move straight forwards or rotate our eyes while moving to become familiar with the surroundings. Sometimes, we would move back to avoid from hitting obstacles. Therefore, the optic flow can be presented in various forms. Specifically, moving straight forward generates radial expansion optic flow (Figure 1a); moving straight backward generates radial contraction optic flow (Figure $1 \mathrm{~b}$ ); if we rotate at a fixed position, a rotation optic flow would be generated (Figure 1c). Of course, sometimes we would laterally move which generates a translation optic flow (Figure 1d). These types of optic flow are generally simple, because they are generated by simple movements. Most of the time in our daily life, our self-movements are complex. For example, we tend to move forward and rotate our eyes to observe the environment, simultaneously. In this situation, a complex spiral expansion optic flow would be generated that is the combination of expansion radial and rotation optic flow (Figures 1e and 1f).

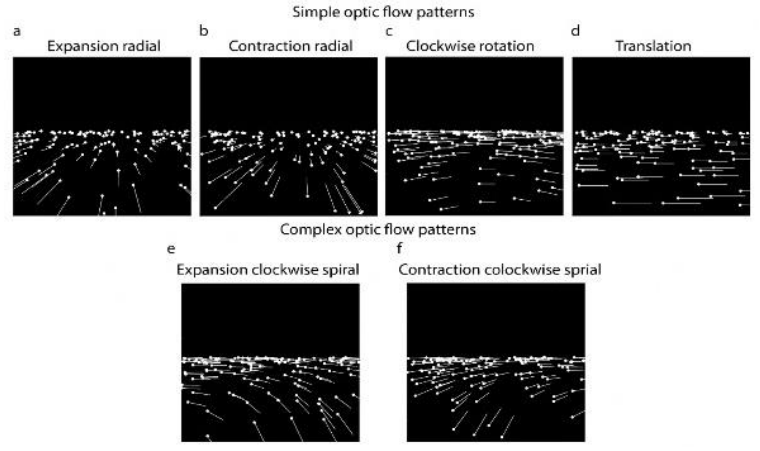

Figure 1. Different types of optic flow patterns. (a) expansion radial optic flow that is generated when observers are moving straight forward; (b) contraction radial optic flow that is generated when observers are moving straight backward; (c) clockwise rotation optic flow that is generated when observers are rotating around the vertical axis clockwise; (d) translation optic flow that is generated when observers are moving laterally to the left or right; (e) expansion clockwise spiral optic flow that is generated when observers are moving straight forward and rotating clockwise; (f) contraction clockwise spiral optic flow that is generated when observers are moving backward and rotating clockwise. This figure was from Sun (2021). 
Additionally, thousands of studies have been conducted and revealed that optic flow plays great roles in many behaviors, such as control of stance (Lim et al., 2018; Piras et al., 2018) and speed (Baumberger et al., 2000; Pretto \& Chatziastros, 2006; Prokop et al., 1997), perception of spatial depth (Li et al., 2016; Simpson, 1993; Wexler \& Van Boxtel, 2005)) and path (Li \& Cheng, 2011; Raudies \& Neumann, 2013; Warren et al., 1991), and the heading (i.e., self-motion direction) perception (Li et al., 2002; Sun et al., 2020; van den Berg, 1992; Warren et al., 2001).

Because of the diversity and the importance of optic flow in our daily behaviors, the neural bases of the optic flow processing attract many researchers' interest. Since now, many cortical areas in human brains have been revealed in the processing of optic flow, e.g., V3a (Bartels et al., 2008; Cardin et al., 2012; Fischer et al. 2012; Kuai et al., 2020; et al., 2013; Strong et al., 2017; Wall et al., 2008; Wall \& Smith, 2008), V3b/KO (Dupont et al., 1997; Kuai, et al., 2020; Wall \& Smith 2008), V7 (Cardin et al., 2012; Ohlendorf et al., 2008), MT+ (MT, MST) (Dukelow et al., 2001; Morrone et al., 2000; Wall et al., 2008), VIP (Field et al., 2020; Furlan et al., 2014; Pitzalis et al., 2013; Wall \& Smith, 2008), CSv (Field et al., 2015; Furlan et al., 2014; Wall \& Smith, 2008; Pitzalis et al., 2013), and p2vc (Fischer et al., 2012; Furlan et al., 2014; Furlan \& Smith, 2016; Pitzalis et al., 2013). In the current article, I mainly reviewed the studies focusing on the area $\mathrm{MT}+$, and proposed the future research direction for the area MT+.

\section{AREA MT+ (MT AND MST) IN HUMAN BRAIN}

Previous studies with the help of PET, histological and fMRI techniques have revealed that area MT+ in the human brain is similar to area MT+ in the monkey brain.

Morrone et al (2000) first divided human MT+ into MST and MT based on their selectivity to the motion patterns. Specifically, area MT tends to respond to the translation 2D global motion patterns, while area MST prefers to respond to the rotation and radial 2D global motion. However, Wall et al (2008) set up an experiment and found some different results. In their experiment, they showed two patterns to the observers while monitoring the BOLD signal using the fMRI techniques. The patterns they presented to the observers were either the same (both are radial or rotation) or different (radial vs. rotation). The results showed that both radial and rotation motion patterns elicited higher BOLD signals suggesting that areas MT and MST in the human brain respond to both the radial and rotation motion patterns.
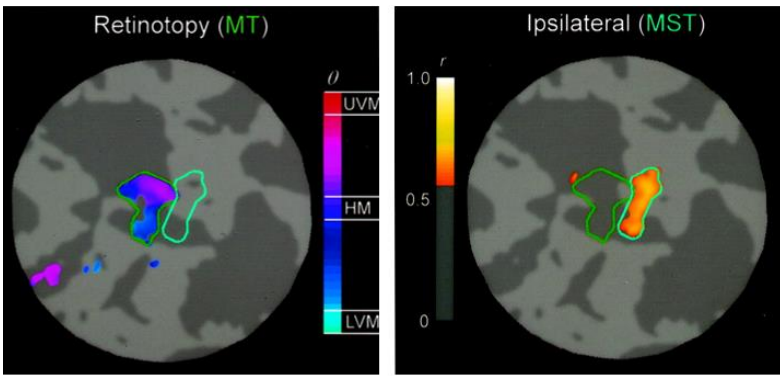

Figure 2. Relative positions of area MT and MST in human brain. The figure is from Huk, Dougherty, \& Heeger (2002).

Dukelow et al (2001) divided MT+ area into MT and MST based on their different receptive visual field features. To be more specific, they discovered that area MT was stimulated by the motion patterns presented in the contralateral visual field. By contrast, area MST responds to the motion patterns presented in both the contra and ipsilateral visual fields. For example, area MT in the left brain prefers to be activated by the motion stimuli in the right eye, but area MST prefers to be activated by the motion stimuli in both eyes. This finding is generally accepted and is always used to locate area MT and area MST in a human brain now (also see Huk, et al., 2002).

\section{QUESTIONS IN THE PREVIOUS STUDIES}

When I was reviewing the previous studies about the area of MT+ in the human brain, I found several questions. First of all, some studies generally took 2D motion patterns as optic flow (e.g., Dukelow et al., 2001; Greenlee, 2000; Giaschi et al., 2007; Koyama et al., 2005; Pitzalis et al., 2010; Rutschmann et al., 2000; Smith et al., 2006; Strong et al., 2017; Wall et al., 2008; Wall \& Smith, 2008; Uesaki \& Ashida, 2015). However, optic flow and 2D motion patterns are different. Specifically, optic flow is the projection of objects as we are moving in the world and containing rich 3D structure information, such as motion parallax or speed gradient (for example, Koenderink, 1986; Longuet-Higgins \& Prazdny, 1980). However, 2D motion pattern is one kind of global motion pattern in which all dots are randomly positioned on a vertical plane and move with a constant speed or a constant acceleration. In this case, no speed gradient or motion parallax is in $2 \mathrm{D}$ motion pattern, which means that no $3 \mathrm{D}$ information was provided to the observer. However, so far, the activities of area MT+ to $2 \mathrm{D}$ global motion patterns and optic flow stimuli were tested separately. i.e., there was no single study in which $2 \mathrm{D}$ motion patterns and optic flow stimuli were tested simultaneously, leading to the question: whether the brain areas that respond to optic flow and 2D global 
motion patterns show unique responses specific to optic flow stimuli.

Additionally, some human-fMRI studies found that area $\mathrm{MT}+$ in the human brain responds to different types of motion patterns differently (Giaschi et al., 2007; Strong et al., 2017; Wall et al., 2008); while some studies found that area MT+ could not do it (Pitzalis et al., 2013). This might due to the former studies adopted a more sophisticated data analysis method, i.e., MVPA analysis; whereas, the other studies only conducted the simple GLM analysis. Compared with GLM analysis, MVPA could reveal the pattern differences between different experimental conditions or stimuli. The different results due to the different data analysis methods lead to the problem of whether human MT+ could differentiate different optic flow patterns remains unclear.

Furthermore, neurophysiological studies (e.g., intracranial electrode technology) have recorded the activities of signal neurons to different types of optic flow and the 2D motion pattern (e.g., Duffy \& Wurtz, 1991a, 1991b), and the information in the motion stimuli (Duffy \& Wurtz, 1997), but no human study investigate that how the individual neurons in area MT+ of human brain respond to optic flow and 2D motion patterns, and the information in the motion stimuli.

Lastly, recent studies about the heading perception with optic flow proposed that heading perception is consistent with the Bayesian account, meaning that the perceived heading biased toward the prior information (e.g., Xing \& Saunders, 2016). Recent monkey neurophysiological studies have figure out the distribution of prior information with intracranial electrode technology ( $\mathrm{Gu}$ et al., 2010). By contrast, the prior information used by human participants is still on the theory.

\section{FUTURE DIRECTIONS}

With the above-mentioned problems in the previous studies, the future work can focus on the following questions:

A series of studies should be conducted in which optic flow and 2D motion patterns can be simultaneously examined so that we can figure out whether the neural basis of 2D motion pattern and optic flow different or not. If they are different, then we can prove the specificity of optic flow processing.

Meanwhile, a more direct research method, e.g., intracranial electrode technology (Campbell \& Wu, 2018), can be applied to the human participants. With this kind of research technology, we can directly reveal the activity features of the human MT+ area to optic flow and solve the disputes because of different data analysis methods.
Furthermore, in the future, with the help of the intracranial electrode technology, we can directly examine whether the neural activities of human MT+ can be modulated by the 3D structure information, e.g., speed gradient. Solving this problem not only helps us reveal the homogeneity of the monkey brain and human brain, but also provides evidence for the specificity of optic flow processing.

Importantly, intracranial electrode technology can also reveal the distribution and selectivity of different MT+ neurons. With the signal neuron data, we can figure out the prior information that we used to modulate our heading perception, providing evidence for the Bayesian account of heading perception.

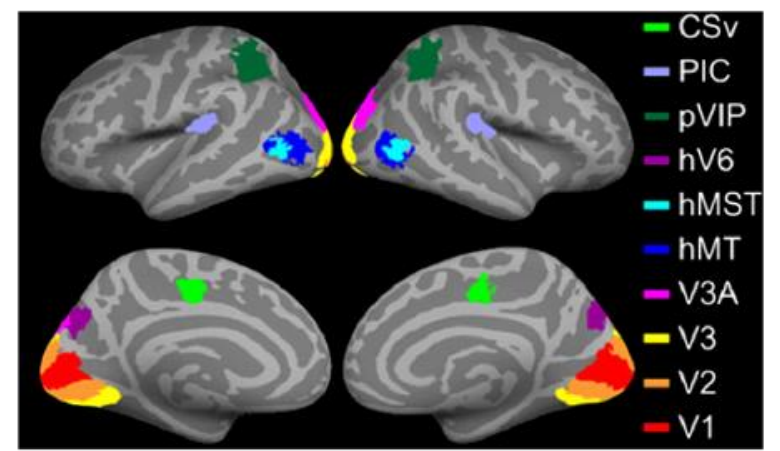

Figure 3 Cortical areas that respond to optic flow and $2 \mathrm{D}$ global motion patterns. Human studies revealed that V3a, V3b/KO, V6, V7, VIP, MT+(hMT/hMST), CSv, $\mathrm{PCi} / \mathrm{Pc} / \mathrm{PcM}, \mathrm{PEc}$, and $\mathrm{p} 2 \mathrm{vc}$ respond to global motion patterns. The figure is edited from Smith et al., (2018).

Lastly, there are many different types of cortical areas in human brain that also respond to optic flow and global motion patterns (Figure 3; please see Greenlee, 2000 for a review). How these cortical areas connected with area $\mathrm{MT}+$, or the information is transformed in these cortical areas is also an important and interesting question.

\section{CONCLUSIONS}

In the current paper, I reviewed the studies about the area MT+ (MST and MT) in human brains. I listed the research questions in the previous studies and provided several research directions to our following researchers. After answering the above questions, we can have a better understanding of optic flow processing, and provide powerful evidence for a long-existing dispute in the area of visual perception and action: what we see is 2D motion pattern or optic flow.

\section{REFERENCES}

[1] Accornero, N., Capozza, M., Rinalduzzi, S., \& Manfredi, G. W. (1997). Clinical multisegmental posturography: age-related changes in stance control. Electroencephalography and Clinical 
Neurophysiology/Electromyography and Motor Control, 105(3), 213-219.

[2] Bartels, A., Zeki, S., \& Logothetis, N. K. (2008). Natural vision reveals regional specialization to local motion and to contrast-invariant, global flow in the human brain. Cerebral Cortex, 18(3), 705717.

[3] Baumberger, B., Flückiger, M., \& Roland, M. (2000). Walking in an environment of moving ground texture. Japanese Psychological Research, 42(4), 238-250.

[4] Campbell, A., \& Wu, C. (2018). Chronically implanted intracranial electrodes: tissue reaction and electrical changes. Micromachines, 9(9), 430.

[5] Cardin, V., Hemsworth, L., \& Smith, A. T. (2012). Adaptation to heading direction dissociates the roles of human MST and V6 in the processing of optic flow. Journal of Neurophysiology, 108, 794801.

[6] Collins, J. J., \& De Luca, C. J. (1995). The effects of visual input on open-loop and closed-loop postural control mechanisms. Experimental Brain Research, 103(1), 151-163.

[7] Crowell, J. A., \& Banks, M. S. (1993). Perceiving heading with different retinal regions and types of optic flow. Perception \& Psychophysics, 53(3), 325-337.

[8] Duffy, C. J., \& Wurtz, R. H. (1991a). Sensitivity of MST neurons to optic flow stimuli. I. A continuum of response selectivity to large-field stimuli. Journal of neurophysiology, 65(6), 1329-1345.

[9] Duffy, C. J., \& Wurtz, R. H. (1991b). Sensitivity of MST neurons to optic flow stimuli. II. Mechanisms of response selectivity revealed by small-field stimuli. Journal of neurophysiology, 65(6), 13461359.

[10] Duffy, C. J., \& Wurtz, R. H. (1997). Medial superior temporal area neurons respond to speed patterns in optic flow. Journal of Neuroscience, 17(8), 2839-2851.

[11] Dukelow, S. P., DeSouza, J. F., Culham, J. C., van den Berg, A. V., Menon, R. S., \& Vilis, T. (2001). Distinguishing subregions of the human MT+ complex using visual fields and pursuit eye movements. Journal of Neurophysiology, 86(4), 1991-2000.

[12] Dupont, P., De Bruyn, B., Vandenberghe, R., Rosier, A. M., Michiels, J., Marchal, G., ... \& Orban, G. A. (1997). The kinetic occipital region in human visual cortex. Cerebral cortex (New York, NY: 1991), 7(3), 283-292.

[13] Field, D. T., Biagi, N., \& Inman, L. A. (2020). The role of the ventral intraparietal area (VIP/pVIP) in parsing optic flow into visual motion caused by self-motion and visual motion produced by objectmotion. NeuroImage, 116679.

[14] Fischer, E., Bülthoff, H. H., Logothetis, N. K., \& Bartels, A. (2012). Visual motion responses in the posterior cingulate sulcus: a comparison to V5/MT and MST. Cerebral Cortex, 22(4), 865-876.

[15] Furlan, M., \& Smith, A. T. (2016). Global motion processing in human visual cortical areas V2 and V3. Journal of Neuroscience, 36(27), 7314-7324.

[16] Furlan, M., Wann, J. P., \& Smith, A. T. (2014). A representation of changing heading direction in human cortical areas pVIP and CSv. Cerebral Cortex, 24(11), 2848-2858.

[17] JGiaschi, D., Zwicker, A., Young, S. A., \& Bjornson, B. (2007). The role of cortical area $\mathrm{V} 5 / \mathrm{MT}+$ in speed-tuned directional anisotropies in global motion perception. Vision research, 47(7), 887-898.

[18] Gibson, J. J. (1950). The perception of the visual world. Boston, MA: Houghton Mifflin.

[19] Greenlee, M. W. (2000). Human cortical areas underlying the pereption of optic flow: brain imaging studies. International Review of Neurobiology, 269-292.

[20] Gu, Y., Fetsch, C. R., Adeyemo, B., DeAngelis, G. C., \& Angelaki, D. E. (2010). Decoding of MSTd population activity accounts for variations in the precision of heading perception. Neuron, 66(4), 596-609.

[21] Huk, A. C., Dougherty, R. F., \& Heeger, D. J. (2002). Retinotopy and functional subdivision of human areas MT and MST. Journal of Neuroscience, 22(16), 7195-7205.

[22] Koenderink, J. J. (1986). Optic flow. Vision Research, 26(1), 161-179.

[23] Koyama, S., Sasaki, Y., Andersen, G. J., Tootell, R. B., Matsuura, M., \& Watanabe, T. (2005). Separate processing of different global-motion structures in visual cortex is revealed by FMRI. Current Biology, 15(22), 2027-2032.

[24] Kuai, S. G., Shan, Z., Chen, J., Xu, Z. X., Li, J. M., Field, D. T., \& Li, L. (2020). Integration of motion and form cues for the perception of self-motion in the human brain. Journal of Neuroscience, 40(5), 1120-1132. 
[25] Larish, J. F., \& Flach, J. M. (1990). Sources of optical information useful for perception of speed of rectilinear self-motion. Journal of Experimental Psychology: Human Perception and Performance, 16(2), 295.

[26] Li, L., \& Cheng, J. (2011). Visual control of steering toward a goal. Journal of Vision, 11(11), 914 [Abstract].

[27] Li, L., Peli, E., \& Warren, W. H. (2002). Heading perception in patients with advanced retinitis pigmentosa. Optometry \& Vision Science, 79(9), 581-589.

[28] Li, J., Lindemann, J. P., \& Egelhaaf, M. (2016). Peripheral processing facilitates optic flow-based depth perception. Frontiers in Computational Neuroscience, 10, 111.

[29] Lim, Y. H., Lee, H. C., Falkmer, T., Allison, G. T., Tan, T., Lee, W. L., \& Morris, S. L. (2018). Effect of optic flow on postural control in children and adults with autism spectrum disorder. Neuroscience, 393, 138-149.

[30] Longuet-Higgins, H. C., \& Prazdny, K. (1980). The interpretation of a moving retinal image. Proceedings of the Royal Society of London. Series B. Biological Sciences, 208(1173), 385-397.

[31] Morrone, M. C., Tosetti, M., Montanaro, D., Fiorentini, A., Cioni, G., \& Burr, D. C. (2000). A cortical area that responds specifically to optic flow, revealed by fMRI. Nature Neuroscience, 3(12), 1322-1328.

[32] Ohlendorf, S., Sprenger, A., Speck, O., Haller, S., \& Kimmig, H. (2008). Optic flow stimuli in and near the visual field centre: a group fMRI study of motion sensitive regions. PloS One, 3(12), e4043.

[33] Peuskens, H., Sunaert, S., Dupont, P., Van Hecke, P., \& Orban, G. A. (2001). Human brain regions involved in heading estimation. Journal of Neuroscience, 21(7), 2451-2461.

[34] Piras, A., Raffi, M., Perazzolo, M., \& Squatrito, S. (2018). Influence of heading perception in the control of posture. Journal of Electromyography and Kinesiology, 39, 89-94.

[35] Pitzalis, S., Sdoia, S., Bultrini, A., Committeri, G., Di Russo, F., Fattori, P., ... \& Galati, G. (2013). Selectivity to translational egomotion in human brain motion areas. PloS One, 8(4), e60241.

[36] Pitzalis, S., Sereno, M. I., Committeri, G., Fattori, P., Galati, G., Patria, F., \& Galletti, C. (2010). Human V6: the medial motion area. Cerebral Cortex, 20(2), 411-424.
[37] Pretto, P., \& Chatziastros, A. (2006). Changes in optic flow and scene contrast affect the driving speed. In Driving Simulation Conference Europe (DSC Europe 2006) (pp. 263-272). Institut National de Recherche sur les Transports et Leur Sécurité.

[38] Prokop, T., Schubert, M., \& Berger, W. (1997). Visual influence on human locomotion modulation to changes in optic flow. Experimental Brain Research, 114(1), 63-70.

[39] Raudies, F., \& Neumann, H. (2013). Modeling heading and path perception from optic flow in the case of independently moving objects. Frontiers in Behavioral Neuroscience, 7, 23.

[40] Rutschmann, R. M., Schrauf, M., \& Greenlee, M. W. (2000). Brain activation during dichoptic presentation of optic flow stimuli. Experimental Brain Research, 134(4), 533-537.

[41] Simpson, W. A. (1993). Optic flow and depth perception. Spatial Vision, 7(1), 35-75.

[42] Smith, A. T., Greenlee, M. W., Singh, K. D., Kraemer, F. M., \& Hennig, J. (1998). The processing of first-and second-order motion in human visual cortex assessed by functional magnetic resonance imaging (fMRI). Journal of Neuroscience, 18(10), 3816-3830.

[43] Smith, A. T., Wall, M. B., Williams, A. L., \& Singh, K. D. (2006). Sensitivity to optic flow in human cortical areas MT and MST. European Journal of Neuroscience, 23(2), 561-569.

[44] Smith, A. T., Beer, A. L., Furlan, M., \& Mars, R. B. (2018). Connectivity of the cingulate sulcus visual area $(\mathrm{CSv})$ in the human cerebral cortex. Cerebral cortex, 28(2), 713-725.

[45] Strong, S. L., Silson, E. H., Gouws, A. D., Morland, A. B., \& McKeefry, D. J. (2017). A direct demonstration of functional differences between subdivisions of human V5/MT+. Cerebral Cortex, 27(1), 1-10.

[46] Sun, Q., Zhang, H., Alais, D., \& Li, L. (2020). Serial dependence and center bias in heading perception from optic flow. Journal of Vision, 20(10), 1-15.

[47] Tootell, R. B., Mendola, J. D., Hadjikhani, N. K., Ledden, P. J., Liu, A. K., Reppas, J. B., ... \& Dale, A. M. (1997). Functional analysis of V3A and related areas in human visual cortex. Journal of Neuroscience, 17(18), 7060-7078.

[48] Uesaki, M., \& Ashida, H. (2015). Optic-flow selective cortical sensory regions associated with 
self-reported states of vection. Frontiers in Psychology, 6(775), 1-9.

[49] van den Berg, A. V. (1992). Robustness of perception of heading from optic flow. Vision Research, 32(7), 1285-1296.

[50] Van Oostende, S., Sunaert, S., Van Hecke, P., Marchal, G., \& Orban, G. A. (1997). The kinetic occipital (KO) region in man: an fMRI study. Cerebral Cortex (New York, NY: 1991), 7(7), 690701.

[51] Wall, M. B., Lingnau, A., Ashida, H., \& Smith, A. T. (2008). Selective visual responses to expansion and rotation in the human MT complex revealed by functional magnetic resonance imaging adaptation. European Journal of Neuroscience, 27(10), 27472757.

[52] Wall, M. B., \& Smith, A. T. (2008). The representation of egomotion in the human brain. Current Biology, 18(3), 191-194.

[53] Warren, W. H., Blackwell, A. W., Kurtz, K. J., Hatsopoulos, N. G., \& Kalish, M. L. (1991). On the sufficiency of the velocity field for perception of heading.

[54] Warren, W. H., Kay, B. A., Zosh, W. D., Duchon, A. P., \& Sahuc, S. (2001). Optic flow is used to control human walking. Nature neuroscience, 4(2), 213-216.

[55] Warren, W. H., Mestre, D. R., Blackwell, A. W., \& Morris, M. W. (1991). Perception of circular heading from optical flow. Journal of Experimental Psychology: Human Perception and Performance, 17(1), 28.

[56] Warren, W. H., Morris, M. W., \& Kalish, M. (1988). Perception of translational heading from optical flow. Journal of Experimental Psychology: Human Perception and Performance, 14(4), 646660.

[57] Xing, X., \& Saunders, J. (2016). Center bias in perceived heading from optic flow. Journal of Vision, 16(12), 884-884. 